\title{
Microstates of a Neutral Black Hole in M Theory
}

\author{
Roberto Emparan ${ }^{1,2, *}$ and Gary T. Horowitz ${ }^{3, \dagger}$ \\ ${ }^{1}$ Institució Catalana de Recerca i Estudis Avançats (ICREA) \\ ${ }^{2}$ Departament de Física Fonamental, Universitat de Barcelona, Diagonal 647, E-08028 Barcelona, Spain \\ ${ }^{3}$ Department of Physics, University of California, Santa Barbara, California 93106-9530, USA
}

(Received 24 July 2006; published 5 October 2006)

\begin{abstract}
We consider vacuum solutions in $\mathrm{M}$ theory of the form of a five-dimensional Kaluza-Klein black hole cross $T^{6}$. In a certain limit, these include the five-dimensional neutral rotating black hole (cross $T^{6}$ ). From a type-IIA standpoint, these solutions carry D0 and D6 charges. We show that there is a simple D-brane description which precisely reproduces the Hawking-Bekenstein entropy in the extremal limit, even though supersymmetry is completely broken.
\end{abstract}

It has been known for more than 30 years that black holes have an enormous entropy. String theory has been able to provide a microscopic description of this entropy in certain cases. For example, the original black hole studied by Strominger and Vafa [1] was charged and supersymmetric. The charges of a black hole serve as tags that help identify its microscopic constituents in string theory. In addition, when the solution is supersymmetric, the phase space of the system is drastically constrained and subject to powerful nonrenormalization theorems and state-counting techniques. Neutral black holes, however, carry a minimal set of quantum numbers - mass and angular momentum and so it seems hard to restrict the phase space to a sector which is simple enough to count the microstates.

Nevertheless, we will argue that there exist vacuum black holes in M theory that can be mapped to well-defined bound states of D-branes in string theory, and which, in certain limits, become asymptotically flat black holes. We start with dyonic solutions of five-dimensional KaluzaKlein (KK) theory [2]. Itzhaki showed[3] that in a certain limit, these solutions include the five-dimensional neutral rotating black hole of Myers and Perry [4], which is asymptotically flat. Reversing this procedure, one can view the KK black hole as the Myers-Perry black hole placed at the tip of a Taub-NUT geometry. A similar connection between four- and five-dimensional black holes has been discussed recently for supersymmetric solutions with additional charges and self-dual angular momentum [5]. Here we are considering the simplest case of a black hole with generic $J_{1}$ and $J_{2}$ and no extra charges.

Taking the product with a flat $T^{6}$, we obtain a solution to M theory, whose type-IIA reduction has D0 and D6 charge. Even in the extremal limit, this black hole is not supersymmetric [6], in accord with the absence of supersymmetric bound states of D0 and D6 branes. There are, however, nonsupersymmetric, quadratically stable, D0D6 bound states [7], and these will serve as a basis to our microscopic picture. We will provide a simple string description that exactly reproduces the entropy and mass of the extremal black hole.
The entropy of some nonsupersymmetric, extremal black holes has been reproduced before [8]. However, that required black holes with four charges (in four dimensions) while we have only two. More importantly, unlike previous examples our solutions are pure vacuum in higher dimensions. The entropy of neutral black holes can be understood in terms of a correspondence principle [9], but that does not reproduce the precise coefficient. To our knowledge, this is the first time that the entropy of a neutral black hole has been reproduced exactly. Earlier work attempting to obtain the precise entropy of asymptotically flat neutral black holes by different means include $[10,11]$. Previous attempts at providing a microscopic description of D0-D6 black holes include [12,13].

We begin by reviewing the KK black holes (for a detailed description, see [2]). These black holes are characterized (in four dimensions) by their mass $M$, angular momentum $J$, and electric and magnetic charges $Q$ and $P$. They satisfy the inequality

$$
2 G_{4} M \geq\left(Q^{2 / 3}+P^{2 / 3}\right)^{3 / 2},
$$

which, at slow rotation $G_{4} J<P Q$, is saturated in the extremal limit independently of $J$. When $P=Q$ and $J=$ 0 the four-dimensional geometry becomes exactly the same as the Reissner-Nordstrom black hole.

In the five-dimensional vacuum solution, let $y$ be the compact KK dimension, $y \equiv y+2 \pi R$. This circle is fibered over two-spheres in the base. Since $S^{1}$ bundles over $S^{2}$ are labeled by an integer, the magnetic charge must be quantized in terms of the radius $R$. The electric charge is also quantized, since it corresponds to momentum in the $y$ direction. More precisely,

$$
Q=\frac{2 G_{4} N_{0}}{R}, \quad P=\frac{N_{6} R}{4}
$$

for integers $N_{0}$ and $N_{6}$ (the reason for this notation will become clear below).

The five-dimensional interpretation of these solutions is quite interesting. In the absence of magnetic charge, the horizon has topology $S^{1} \times S^{2}$, where $S^{1}$ is the KK circle, 
and the solution is a black string boosted along $y$. However, the topology changes when $P \neq 0$. If $N_{6}=1$, the $y$ circle and spherical $S^{2}$ combine into a topological $S^{3}$. In the extremal limit with $Q=0$ and $J=0$, the solution becomes the KK monopole. The geometry can be described as a "cigar" fibered on the orbital $S^{2}$.

If we add electric charge or energy above extremality, we find a finite black hole horizon at the tip of the cigar. So magnetically charged KK black holes are five-dimensional black holes with horizon topology $S^{3}$, localized inside a Taub-NUT geometry. The electric charge does not correspond to a boost, but rather to rotation of the black hole aligned with the KK circle. A component of the rotation that is not aligned with the five-dimensional fiber gives rise to four-dimensional rotation.

If the size of the black hole is much smaller than the KK radius $R$, then finite-size effects become negligible and we recover the five-dimensional Myers-Perry black hole, as explained in [3]. In this limit, the four-dimensional mass is dominated by the mass of the $\mathrm{KK}$ monopole, and the excitation energy above the KK monopole is equal to the mass of the five-dimensional black hole. The angular momenta in five dimensions are related to the electric charge and four-dimensional angular momentum as $J_{1}+J_{2}=$ $N_{0} N_{6}^{2}$ and $J_{1}-J_{2}=2 J N_{6}$.

The identification with a five-dimensional black hole is a local one. Globally, the asymptotic spatial geometry is actually the orbifold $\mathbf{R}^{4} / \mathbf{Z}_{N_{6}}$. So only configurations with $N_{6}=1$ give rise to globally asymptotically flat solutions. When $N_{6}>1$ the black hole sits at the tip of a conical space.

The entropy of the KK black hole is particularly simple in the extremal limit [13],

$$
S=\frac{A_{(4)}}{4 G_{4}}=2 \pi \sqrt{\frac{P^{2} Q^{2}}{G_{4}^{2}}-J^{2}}=2 \pi \sqrt{\frac{N_{0}^{2} N_{6}^{2}}{4}-J^{2} .}
$$

This is independent of the circle radius $R$, so it also corresponds to the entropy of the extremal Myers-Perry black hole after the limit of infinite radius $R$ is taken. It was noted in $[13,14]$ that the entropy depends only on the integer normalized charges. This is a strong indication that a microscopic counting of the states is possible.

In order to count the microstates of the KK black hole, we take the product with $T^{6}$ [with volume $(2 \pi)^{6} V_{6}$ ] and view it as a vacuum solution in M theory with the KK circle being the $\mathrm{M}$ theory circle. By the usual relation between $\mathrm{M}$ theory and type-IIA string theory, $R=g l_{s}$. The electric and magnetic charges now correspond to D0 and D6branes, and $N_{0}$ and $N_{6}$ are simply the net number of Dbranes. The quantization condition (2) can now be written

$$
Q=2 G_{4} M_{0} N_{0}, \quad P=2 G_{4} M_{6} N_{6}
$$

where the masses of individual D0 and D6-branes are

$$
M_{0}=\frac{1}{g l_{s}}, \quad M_{6}=\frac{V_{6}}{g l_{s}^{7}},
$$

and $G_{4}=g^{2} l_{s}^{8} / 8 V_{6}$. So the mass of our extremal black hole is

$$
M=\left[\left(M_{0} N_{0}\right)^{2 / 3}+\left(M_{6} N_{6}\right)^{2 / 3}\right]^{3 / 2} .
$$

To reproduce this mass and the entropy formula (3) we will pass to a $T$ dual configuration where the microscopic description becomes more transparent. For simplicity we consider first the case without four-dimensional rotation, $J=0$, and will discuss $J \neq 0$ near the end.

We first recall the situation for the supersymmetric fourcharge black holes in Type II string theory compactified on $T^{6}$. There are many possible choices for the charges, all related by $U$ duality. For our purposes, the most useful is in terms of four stacks of D3-branes [15]. Any two stacks intersect over a line, and all four intersect at a point. The orientation of the first three stacks can be chosen arbitrarily, but to preserve supersymmetry, the orientation of the last set of D3-branes is then fixed. We are interested in the case where the number of branes in each stack is the same, say $N$. The moduli of the $T^{6}$ then remain constant, and the solution reduces to the product of $T^{6}$ and extreme Reissner-Nordstrom,

$$
d s^{2}=-\left(1+\frac{r_{1}}{r}\right)^{-2} d t^{2}+\left(1+\frac{r_{1}}{r}\right)^{2}\left(d r^{2}+r^{2} d \Omega_{2}\right) .
$$

Assuming a square torus with equal size circles and $V_{6}=$ $\left(V_{3}\right)^{2}$, the constant $r_{1}$ is related to the number of 3-branes $N$ via $r_{1}=\frac{g N l_{s}^{4}}{2 V_{3}}$. The mass is

$$
M=\frac{r_{1}}{G_{4}}=\frac{4 N V_{3}}{g l_{s}^{4}},
$$

which is just the mass of the four stacks of $N$ 3-branes wrapped around the torus, and the black hole entropy is

$$
S=\frac{A}{4 G_{4}}=2 \pi N^{2} .
$$

Although the explicit counting of states for supersymmetric four-dimensional black holes is easier to carry out with a different choice of charges [16], the fact that it reproduces (9) and is related by $U$ duality ensures that the D3branes also contain precisely the right number of states (at large $N$ ) to reproduce the black hole entropy. Furthermore, since the entropy is independent of the moduli of the torus, it seems clear that the states are associated with the intersection point of the branes.

Bound states of four D0-branes and four D6-branes were described by Taylor in terms of a gauge theory configuration on the world volume on the 6-brane [7]. He pointed out that after applying $T$ duality along three cycles on the torus, this configuration was equivalent to four D3-branes in a configuration very similar to the one described above. However, there are two important differences. The orientations correspond to broken supersymmetry, and the branes are wrapping the diagonals of the torus. To be explicit, consider first a square $T^{2}$ with coordinates $\left(x_{1}\right.$, $x_{2}$ ). The two diagonals are given by $x_{2}= \pm x_{1}$ which we will call the + and - cycle. If we orient the cycles so that 
$x_{2}$ always increases, then a configuration of two strings wrapping both diagonals has net winding number two around $x_{2}$ and zero winding around $x_{1}$ (see Fig. 1). Now view $T^{6}$ as the product of three $T^{2}$, s, with coordinates $\left(x_{1}\right.$, $\left.x_{2}\right),\left(x_{3}, x_{4}\right)$ and $\left(x_{5}, x_{6}\right)$, respectively. The 3-branes are all wrapped around one diagonal of each $T^{2}$ and oriented so that the even coordinates always increase. So the configuration can be labeled by specifying which of the diagonals is wrapped on each $T^{2}$. If we $T$ dualize in the 2,4 , 6 directions, the configuration dual to the four D0-branes and four D6-branes is

$$
(+++),(+--),(-+-),(--+),
$$

where the first entry corresponds to the first torus, etc. By construction, each brane wraps the cycle (246) once, and since each entry has an even number of minus signs, each brane also wraps the cycle (135) once. It is easy to check that the net winding about any other 3-cycle [such as (146)] is zero. So even though there are four 3-branes, the net nonzero charges are just (135) and (246), which is what one expects after three $T$ dualities of D0-D6.

If we replace the single 3-brane around each cycle in (10) by $N$, we obtain a configuration with charge $4 N$ around each of the cycles (135) and (246). This reproduces the mass of the black hole. After three $T$ dualities, we get $N_{0}=N_{6}=4 N$ and hence (6) becomes $M=$ $2^{3 / 2} 4 N V_{3} / g l_{s}^{4}$. [Starting with a symmetric $T^{6}$ for the intersecting 3-branes and applying three $T$ dualities results in a torus with volume $V_{6}=l_{s}^{6}$ (and changes the string coupling to $\left.g l_{s}^{3} / V_{3}\right)$. So the 6-brane and 0-brane have equal mass.] This is equal to the mass of the 3-branes since the length of each leg is $\sqrt{2}$ larger than before, so the volume of each 3-brane is $2^{3 / 2}$ greater. The fact that the mass does not saturate a BPS bound is just a reflection of the fact that the branes are wrapping cycles of larger volume.

What about the entropy? At first sight there appears to be a discrepancy. If we make the reasonable assumption that the entropy of the intersecting 3-branes is unaffected by the change in orientation and rotation of the branes we would

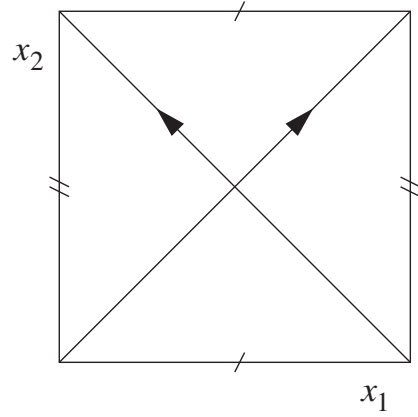

FIG. 1. Branes wrapping the diagonals of a torus. There are two intersection points, at the origin and at the middle of the square. We assume that each intersection contributes a microscopic entropy equal to that of a supersymmetric intersection of branes. expect $S=2 \pi N^{2}$ as in (9). However, since $N_{0}=4 N$ and $N_{6}=4 N$ (and $J=0$ ) the black hole entropy (3) is

$$
S_{\mathrm{bh}}=16 \pi N^{2} \text {, }
$$

which is larger by a factor of 8 . However, rotating the branes increases the number of intersection points (we thank Juan Maldacena for suggesting this). On a $T^{2}$, the diagonals have two intersection points (see Fig. 1). Since the branes have two intersection points on each of the three $T^{2}$, s, there are a total of eight intersection points. The total Hilbert space is a tensor product of the states at each intersection point and hence

$$
S_{\text {branes }}=8 \times 2 \pi N^{2} .
$$

Thus, a simple calculation reproduces the black hole entropy exactly.

It is easy to generalize this to the case of unequal charges (in terms of gauge fields on the 6-brane, this was done in $[13,14])$. The configuration of branes is again given by $(10)$ where \pm now refer to more general cycles than just the diagonal. Let \pm denote the cycles $x_{2}= \pm k x_{1} / l$ for relatively prime integers $k, l$ (and similar cycles on the other two $T^{2}$ 's with the same integers $k, l$ ). The configuration of branes (10) now has charge $4 k^{3}$ along (246) and charge $4 l^{3}$ along (135). The mass of each brane is now $\left(k^{2}+l^{2}\right)^{3 / 2}$ larger just from the increase in area of the three-cycle being wrapped. This agrees with the black hole mass since with $N$ branes wrapped around each of the cycles, $N_{0}=4 k^{3} N$, $N_{6}=4 l^{3} N$ so (6) yields

$$
M_{\mathrm{bh}}=\frac{4 N\left(k^{2}+l^{2}\right)^{3 / 2} V_{3}}{g l_{s}^{4}}=M_{\mathrm{branes}} .
$$

In retrospect, the presence of $3 / 2$ in the exponent of the black hole mass is an indication of a microscopic description in terms of 3-branes.

The entropy also comes out exactly right since the + and - cycles now have $2 k l$ intersection points on each $T^{2}$ (see Fig. 2). So the collection of 3-branes has a total of $(2 k l)^{3}$ intersection points. The entropy is thus

$S_{\text {branes }}=(2 k l)^{3} \times 2 \pi N^{2}=\pi\left(4 N k^{3}\right)\left(4 N l^{3}\right)=\pi N_{0} N_{6}=S_{\text {bh }}$.

The limiting case of a Myers-Perry black hole described above requires only a slight generalization (see Fig. 2). We want to take $R \rightarrow \infty$ keeping $G_{5}, G_{11}, N_{0}$ and $N_{6}$ fixed. In

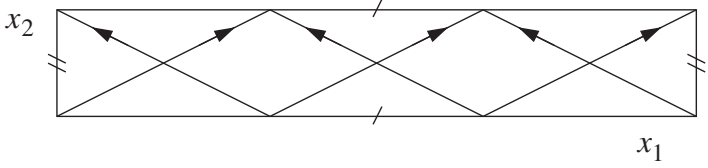

FIG. 2. Generalization to unequal charges and nontrivial moduli. The branes wrap a rational direction $k / l$ of the torus (in the figure, $k=3, l=1$ ), so there are $2 k l$ intersection points on each $T^{2}$. In the limit to the five-dimensional Myers-Perry black hole, the torus shrinks along $x_{2}$. 
the D0-D6 frame, this corresponds to taking $g \rightarrow \infty$ keeping $V_{6}$ fixed in Planck units. Since the 11-dimensional Planck length $l_{p}$ is given by $l_{p}=g^{1 / 3} l_{s}$, when we $T$ dualize along a direction, the new circle has length $\tilde{L} \sim l_{s}^{2} / L \sim$ $g^{-2 / 3}$. Thus, after $T$ duality in the 2, 4, 6 directions, the size of these three circles goes to zero in the limit. (The IIB string coupling remains finite since $\tilde{g} \sim g l_{s}^{3} / L^{3} \sim l_{p}^{3} / L^{3}$.) We again obtain 3-branes wrapping the cycles (10), but they become essentially parallel as we approach the Myers-Perry black hole, all wrapping the (135) cycle with a positive orientation. Since the entropy is moduli independent, the equality between statistical and black hole entropies holds as in (14).

Finally, to allow for $J \neq 0$ we assume that $J$ is evenly distributed among the $(2 k l)^{3}$ intersections of 3-branes so each one carries angular momentum $J_{0}=J /(2 k l)^{3}$. In the $(4,0)$ theory that describes the four-charge system, to account for $J_{0}$ we align the polarization of $J_{0}^{2} / N^{3}$ fermionic left-moving excitations (out of $N$ ) while the right movers remain unexcited. The entropy is then $2 \pi \sqrt{N^{3}\left(N-J_{0}^{2} / N^{3}\right)}$. (This is the nonsupersymmetric ana$\log$ of [17].) Assuming that this applies to each intersection, the mass formula is not modified but the total microscopic entropy becomes

$$
S_{\text {branes }}=(2 k l)^{3} \times 2 \pi \sqrt{N^{4}-J_{0}^{2}}=2 \pi \sqrt{\frac{N_{0}^{2} N_{6}^{2}}{4}-J^{2}} .
$$

This reproduces (3) and hence also the entropy of the extremal rotating Myers-Perry black holes with generic angular momenta.

It seems remarkable that a simple system of D-branes is able to reproduce the mass and entropy of a vacuum black hole which is far from being supersymmetric. It is not clear to us why this is working so well, but it hints at further simplifications that might be possible for neutral black holes. In particular, it is intriguing that we need to use four different sets of branes, even though (from the typeIIA standpoint) there are only two charges. This is reminiscent of earlier suggestions that neutral black holes should be viewed as collections of branes and antibranes $[11,18]$. There is a mysterious duality invariant formula which reproduces the entropy of all nonextremal black holes (including Schwarzschild) in terms of branes and antibranes. It is not yet clear how to derive this in string theory, but the above construction seems a step in the right direction.

Various other open questions remain: (1) We have only considered extreme KK black holes. Can one count the microstates of near-extremal solutions? This would bring us a little closer toward understanding Schwarzschild. (2) Can one reproduce the entropy of KK black holes with $N_{6}=1$ ? The constructions above seem to require that $N_{6} \geq 4$ (actually $N_{6} \gg 4$, since assigning an entropy $2 \pi N^{2}$ to each intersection is justified only for large $N$ ).
(3) Can one replace $T^{6}$ with general Calabi-Yau spaces and still count the entropy? Since mirror symmetry can be viewed as $T$ duality on a $T^{3}$, an initial collection of D0 and D6-branes goes over to a collection of D3-branes under this symmetry. This suggests that the above construction may have a natural generalization. (4) Rather than working with D3-branes, can one understand the entropy of the above black holes directly in $\mathrm{M}$ theory (in terms of gravitons and perhaps branes) or in terms of D6-branes with flux corresponding to D0-branes? This latter case corresponds to counting the number of instantons (of a certain type) in six-dimensional Yang-Mills theory.

We thank the KITP, Santa Barbara for the stimulating program "Scanning New Horizons: GR Beyond 4 Dimensions" where this work was begun. We also thank R. Kallosh, F. Larsen, J. Polchinski, S. Trivedi, and especially J. Maldacena for discussions. G. H. thanks the IAS Princeton for their hospitality. R. E. was supported in part by DURSI No. 2005 SGR 00082, CICYT FPA No. 200404582-C02-02, and EC FP6 program No. MRTN-CT2004-005104. G. H. was supported in part by NSF Grants No. PHY-0244764 and No. PHY-0555669.

*Electronic address: emparan@ub.edu

${ }^{\dagger}$ Electronic address: gary@physics.ucsb.edu

[1] A. Strominger and C. Vafa, Phys. Lett. B 379, 99 (1996).

[2] G. W. Gibbons and D. L. Wiltshire, Ann. Phys. (N.Y.) 167, 201 (1986); 176, 393(E) (1987); D. Rasheed, Nucl. Phys. B454, 379 (1995); F. Larsen, Nucl. Phys. B575, 211 (2000).

[3] N. Itzhaki, J. High Energy Phys. 09 (1998) 018.

[4] R. C. Myers and M. J. Perry, Ann. Phys. (N.Y.) 172, 304 (1986).

[5] D. Gaiotto, A. Strominger, and X. Yin, J. High Energy Phys. 02 (2006) 024.

[6] R. R. Khuri and T. Ortín, Phys. Lett. B 373, 56 (1996).

[7] W. I. Taylor, Nucl. Phys. B508, 122 (1997).

[8] A. Dabholkar, Phys. Lett. B 402, 53 (1997).

[9] G. T. Horowitz and J. Polchinski, Phys. Rev. D 55, 6189 (1997).

[10] R. Argurio, F. Englert, and L. Houart, Phys. Lett. B 426, 275 (1998).

[11] U. H. Danielsson, A. Guijosa, and M. Kruczenski, J. High Energy Phys. 09 (2001) 011.

[12] H. J. Sheinblatt, Phys. Rev. D 57, 2421 (1998).

[13] F. Larsen, hep-th/0002166.

[14] A. Dhar and G. Mandal, Nucl. Phys. B531, 256 (1998).

[15] V. Balasubramanian and F. Larsen, Nucl. Phys. B478, 199 (1996); I. R. Klebanov and A. A. Tseytlin, Nucl. Phys. B475, 179 ( 1996).

[16] J. M. Maldacena and A. Strominger, Phys. Rev. Lett. 77, 428 (1996); C. V. Johnson, R. R. Khuri, and R. C. Myers, Phys. Lett. B 378, 78 (1996).

[17] G. T. Horowitz, D. A. Lowe, and J. M. Maldacena, Phys. Rev. Lett. 77, 430 (1996).

[18] G. T. Horowitz, J. M. Maldacena, and A. Strominger, Phys. Lett. B 383, 151 (1996). 